\title{
Avascular Necrosis of the Femoral Head in Dogs - Retrospective Study
}

\author{
Catarina Borges Cardoso', Sheila Canevese Rahal', Maria Jaqueline Mamprim², Hugo Salvador Oliveira², \\ Alessandra Melchert ${ }^{3}$, Jeniffer Gabriela Figueroa Coris' ${ }^{1}$ \& Luciane dos Reis Mesquita' ${ }^{1}$
}

\begin{abstract}
Background: The avascular necrosis of the femoral head is a development disease caused by ischemic necrosis, which is mainly observed in young dogs. The etiology of the disease remains controversial. The diagnosis requires imaging exams such as MRI and radiographs. Thus, the aim of the current study was to retrospectively assess a population of dogs with avascular necrosis of the femoral head in order to feature the disease, as well as to analyze the radiographic appearance of the lesion at the moment of patient consultation.

Materials, Methods \& Results: The signalment factors of dogs (breed, gender, age and body mass), the affected hind limb, the radiographic appearance of the lesion, the clinical signs at the moment of patient consultation, the time of occurrence and the type of treatment were evaluated. The disease was radiographically classified according to the previously described items. Forty-three cases of avascular necrosis of the femoral head were identified, 97.67\% presented lameness and pain during palpation of the hip joint; and 54.34\%, were 1 week to 4 months old. Females represented $58.13 \%$ of the sample, and $65.11 \%$ of them weighed from 2.6 to $4.9 \mathrm{~kg}$. Based on the radiographic classification, $4.34 \%$ were Grade 1; 32.60\%, Grade $2 ; 8.69 \%$, Grade $3 ; 19.56 \%$, Grade 4 ; and $34.78 \%$, Grade 5 . The femoral head and neck ostectomy was performed in 42 hind limbs (91.30\%); $42.85 \%$ of the dogs reached total functional recovery and $26.19 \%$ required physiotherapy and rehabilitation. Discussion: The present sample was composed of $25 \mathrm{dogs}$, which were 6-to-11-month old at the moment of patient consultation, but 17 dogs were 12-to-36-month old at this time; only one dog was older than 36 months. It may be associated with the non-recognition of clinical signs by the owners, rather than with the late-onset form of the disease. With respect to the breed, pinscher, Yorkshire, poodle, Lhasa apso, pug were most frequently observed. However, 7 dogs were crossbreed. Such group differed from that of a review involving 188 cases encompassing West Highland white terrier, Cairn terrier and poodle as the most commonly affected breeds. No sex predilection was found in a study comprising 188 cases, but in another study comprising $14 \mathrm{dogs}$, female predilection was observed. Likewise, the females represented $58.13 \%$ of cases in the present study; and $41.86 \%$ of the participants were male. The mean body mass of the dogs in the current study was $4.33 \mathrm{~kg}$, which matches the small breed dogs, which are most affected by the disease. Clinical signs of non-weightbearing lameness or an intermittent subtle lameness are common in the avascular necrosis of the femoral head. Lameness of the affected hind limb was observed in $69.76 \%$ of the cases in the present study, but $2.32 \%$ showed no lameness. Occasionally, the avascular necrosis of the femoral head is bilaterally found in $12 \%$ to $16.5 \%$ of the cases. Only 3 out of the 43 cases assessed in the present study were bilateral. Since the lesions were more frequent in Grades 2 and 5, there is less chance of success through the conservative treatment. Thus, the femoral head and neck ostectomy was the surgical procedure performed in 42 hind limbs. The procedure is used to provide pain relief and to reduce the signs of lameness. In conclusion, the population assessed in the present study was composed of small size dogs, mean body mass $4.33 \mathrm{~kg}$, no sex predilection, mostly presenting unilateral lesions and higher Grade 2 and Grade 5 radiographic lesion frequency.
\end{abstract}

Keywords: radiographic, joint, canine, hip. 


\section{INTRODUCTION}

Many disorders such as fractures of the femoral neck, acetabulum or greater trochanter; luxation; dysplasia; and avascular necrosis of the femoral head, also called Legg-Calvé-Perthes disease [6,14], may compromise the coxofemoral joint. The avascular necrosis of the femoral head is a development disease caused by ischemic necrosis, which is mainly observed in young dogs $[1,6,7,17]$.

The etiology of the disease remains controversial, and several causes such as infection, trauma, hereditary, hormonal and metabolic imbalances, anatomical conformation, and vascular abnormalities, among others [1,2,15-19] have been cited in the literature. Histological findings show that there is correlation among disease stage, dog age and clinical sign duration [2].

The palpation of the hip may result in pain during abduction or extension movements, and crepitation and limitation of extension may be observed $[2,6,12,14]$. The hind limb may be shortened, the greater trochanter gets prominent, and there is atrophy of the gluteal and quadriceps muscles $[1,2,6,12]$. In severe cases, the dog only uses the forelimbs to locomotion, there is no weightbearing on the hind limbs $[6,12]$. The diagnosis requires imaging exams such as MRI and radiographs $[6,11,12]$. The radiographic findings in early disease stages show femoral head enlargement due to bone proliferation. The femoral head becomes flattened after 4 months and collapses after 6 months; such condition leads to changes in the pelvic bones, acetabulum, femur and in the tibia [1,12]. The flattening of the femoral head seems to be related to mechanical collapse, asymmetrical growth and to disturbances in endochondral ossification [10].

Thus, the aim of the current study is to retrospectively assess a population of dogs with avascular necrosis of the femoral head in order to feature the disease, as well as to analyze the radiographic appearance of the lesion at the moment of patient consultation.

\section{MATERIALS AND METHODS}

\section{Data analysis}

Data from a period of ten years and 3 months of dogs with avascular necrosis of the femoral head, treated at the Veterinary Hospital of our Veterinary School, were retrospectively analyzed.

\section{Clinical signs and radiographic exams}

The signalment factors of dogs (breed, sex, age and body mass), the affected hind limb, the radiographic appearance of the lesion, the clinical signs at the moment of patient consultation, the time of occurrence and the type of treatment were evaluated.

The disease was radiographically classified according to previously described items [10], as follows: Grade 1- acetabulum and contour of the femoral head and of the femoral neck are apparently normal, and there is wide joint space, decreased density of the femoral head ( 1 or multiple foci) and, sometimes, of the femoral neck; Grade 2- there is femoral head flattening and the presence of multiple density foci of decreased density, the acetabular rim may contain a small spur; Grade 3- there is irregularity in the articular surface of the femoral head and the presence of multiple low density foci, the spur is more prominent in the acetabular rim; Grade 4- there is loss of the normal shape of the femoral head, increased prevalence of areas with decreased density; Grade 5- there is fragmentation of the femoral head with discontinuity of the articular surface, and the acetabular changes are more pronounced than those observed in Grade 4.

\section{Statistical Analysis}

The simple linear regression analysis was used to quantify the relationship among lesion grade, body mass and age. The Spearman's correlation was used to analyze the association between lesion grade and other parameters (gender, breed, affected hind limb, unilateral or bilateral lesion, time of occurrence, and type of treatment). The differences were considered significant at $P<0.05$. In addition, the data were analyzed using descriptive statistics to feature the frequency of numerical variables.

\section{RESULTS}

Forty-three cases ( $n=46$ hind limbs) of avascular necrosis of the femoral head were identified in the present study. Of the breeds recorded, $27.9 \%$ of the dogs were pinscher $(n=12) ; 23.25 \%$ were Yorkshire $(\mathrm{n}=10) ; 11.62 \%$ were poodle $(\mathrm{n}=5) ; 6.97 \%$ were Lhasa apso $(n=3) ; 4.65 \%$ were pug $(n=2) ; 9.30 \%$ consisted of other breeds with only one representative (Chihuahua, Dachshund, Pekingese, and Brazilian terrier); and $16.27 \%$ were crossbreed $(n=7)$. Twenty-five patients were females $(58.13 \%)$ and 18 were males $(41.86 \%)$. According to age group at the moment of 
patient consultation, 25 dogs were 6-to-11-month old (58.13\%), 17 dogs were 12-to-36-month old (39.53\%), and one dog was older than 36 months $(2.32 \%)$. Regarding body mass, $16.27 \%(\mathrm{n}=7)$ of the dogs weighed $1.4-2.5 \mathrm{~kg} ; 65.11 \%(\mathrm{n}=28)$ ranged from 2.6 to $4.9 \mathrm{~kg}$, and $18.60 \%(\mathrm{n}=8)$ weighed $5-7.5 \mathrm{~kg}$.

Lameness of the affected hind limb was observed in $69.76 \%(n=30)$ of the cases; $27.90 \%(n=12)$ of them presented non-weight bearing; and $2.32 \%$ (n $=1$ ), showed no lameness.

Pain in the limbs during palpation of the hip joint was detected in $97.67 \%(n=42)$ of the cases; $62.79 \%(n=27)$ of it was associated with crepitus. Atrophy in the hind limb was identified in $13.95 \%$ (n $=6)$ of the patients, and $46.51 \%(n=20)$ of the dogs showed different degrees of medial patellar luxation.

According to the anamnesis, the clinical signals in the present study were observed between 1 week and 4 months of life in $54.34 \%$ of the cases $(n=25)$; between 5 and 6 months in $26.08 \%(n=12)$ of the cases; and in $19.56 \%(n=9)$ of the cases, the owner did not inform about the disease. In 41 dogs $(93.03 \%)$ the disease was unilateral and in three dogs it was bilateral (6.97\%), two males and one female. Fifty-five 55\% (n $=22$ ) of the unilateral lesions were located in the right hind limb; and 45\% ( $\mathrm{n}=18)$, in the left hind limb.

The radiographic grade of the lesion was not statistically significant in the present study in comparison to other variables in both the simple linear regression and the Spearman's correlation. Thus, the descriptive analysis was done based on the radiographic classification: $4.34 \%$ were Grade $1(n=2) ; 32.60 \%$, Grade $2(n=15)$; $8.69 \%$, Grade $3(n=4) ; 19.56 \%$, Grade $4(n=9)$; and $34.78 \%$, Grade $5(n=16)$ [Figure 1]. In addition, Grade 5 was the most frequent $(n=9 ; 36 \%)$ in lesions with up to 4 months evolution ( $\mathrm{n}=25$ cases), it was followed by Grade $2(n=6 ; 24 \%)$ and by Grade $4(n=6 ; 24 \%)$.
Surgical treatment was performed in 42 hind limbs $(91.30 \%)$ and conservative treatment in one patient (12 months old, unilateral lesion). Total functional recovery of the hind limb was observed in $42.85 \%(n=18)$ of the dogs after surgery, and $26.19 \%(\mathrm{n}=11)$ required physiotherapy and rehabilitation. Partial recovery was observed in $21.42 \%(\mathrm{n}=9)$ of the dogs, and in $4.76 \%$ ( $\mathrm{n}$ $=2$ ) of them another procedure was necessary to remove the remaining portion of the femoral neck, and it resulted in complete recovery. The owner did not return the dog for postoperative follow up in $4.76 \%$ of the cases $(n=2)$.

\section{DISCUSSION}

The clinical signs of the avascular necrosis of the femoral head generally appear between 3 and 13 months of life, the peak incidence occurs at 5 and 8 months; the lameness may be present for several weeks or there may be sudden onset of it [2]. The present sample was composed of $25 \mathrm{dogs}$, which were 6-to-11-month old at the moment of patient consultation, but 17 dogs were 12-to-36-month old at this time; only one dog was older than 36 months. The aforementioned occurrence was also cited in a study comprising $14 \mathrm{dogs}$, wherein $35.71 \%$ of them were older than 12 months at the time they were assessed [9]. It may be associated with the non-recognition of clinical signs by the owners, rather than with the late-onset form of the disease.

The small breed dogs are most often affected by the disease and hereditary factors may be involved in breeds such as miniature poodle, West Highland white terrier, and Manchester terrier [1,6,15,19].

With respect to the breed, pinscher $(n=12)$, Yorkshire $(\mathrm{n}=10)$, poodle $(\mathrm{n}=5)$, Lhasa apso $(\mathrm{n}$ $=3)$, pug $(n=2)$ were most frequently observed in the present study. However, 7 dogs were crossbreed. Such group differed from that of a review involving 188 cases encompassing West Highland white terrier, Cairn terrier and poodle as the most commonly affected
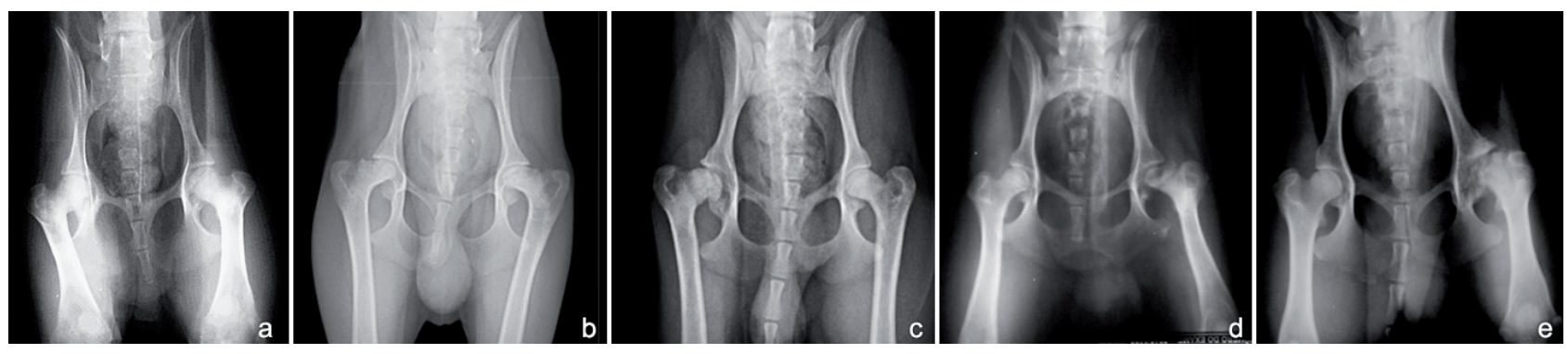

Figure 1. Ventrodorsal radiographic views of the hip joints in dogs with avascular necrosis of femoral head. a- Grade 1: Decreased density of the femoral head; b- Grade 2: Flattening of the femoral head and presence of density foci of decreased density; c- Grade 3: Irregularity of the articular surface of the femoral head and presence of multiple low density foci, spur on the acetabular rim; d- Grade 4: Loss of normal shape of the femoral head, increased prevalence of the areas with decreased density; e- Grade 5: Fragmentation of the femoral head and discontinuity of the articular surface. 
breeds [8]. On the other hand, in a study comprising $35 \mathrm{dogs}, 60 \%$ of them were Yorkshire terrier [13]; and in another study comprising $14 \mathrm{dogs}$, the incidence of the disease was higher in Pekingese and Terrier breeds [9]. The differences may be associated with the owner's preference for some breeds or even with economic factors.

No sex predilection was found in a study comprising 188 cases [8], but in another study comprising 14 dogs, female predilection was observed [9]. Likewise, the females represented $58.13 \%$ of cases in the present study; and $41.86 \%$ of the participants were male. The mean body mass of the dogs in the present study was $4.33 \mathrm{~kg}$, which matches the small breed dogs, which are most affected by the disease $[1,18]$.

Clinical signs of non-weight-bearing lameness or an intermittent subtle lameness are the most frequently observed in the avascular necrosis of the femoral head $[1,12]$. Lameness of the affected hind limb was observed in $69.76 \%$ of the cases in the present study, but $2.32 \%$ showed no lameness. Additionally, $46.51 \%$ of the dogs showed different patellar luxation degrees, and it may have contributed to the lameness cases. Pain in the limbs during palpation of the hip joint was detected in $97.67 \%$ of the cases; $62.79 \%$ of it was associated with crepitus. In general, the pain may get worse during hip joint abduction [12]. On the other hand, atrophy in the hind limb was identified in only $13.95 \%$ of the patients, in the present study.

Occasionally, the avascular necrosis of the femoral head is bilaterally found in $12 \%$ to $16.5 \%$ of the cases [18]. Only 3 out of the 43 cases assessed in the present study were bilateral $(6.97 \%)$. They were Pinscher and Yorkshire breeds, up to 14 months old and presented different grades between hind limbs.

Based on the radiographic findings, $34.78 \%$ of the lesions were classified as Grade 5, 32.60\% were Grade 2, $19.56 \%$ were Grade 4, 8.69\% were Grade 3, and $4.34 \%$ were Grade 1 . Since the lesions were more frequent in Grades 2 and 5, there is less chance of success through the conservative treatment. In addition, Grade 5 was the most frequent $(36 \%)$ in lesions with up to 4 months evolution, followed for Grades 2 and 4 with the same percentages (24\%). A study comprising seven dogs with monolateral avascular necrosis of the femoral head found that the radiographic areas presenting decreased density in Grades 2 and 3 do not alter the global mineral content of the scanned region in dual-energy X-ray absorptiometry scans [4].

Conservative and surgical treatment have been used $[1,9,14,18]$. The conservative treatment includes rest, controlled exercise, analgesic and anti-inflammatory medication, external coaptation, and physiotherapy $[2,3,9,12]$. It was only performed in one case, in the present study through anti-inflammatory and chondroprotective medication and acupuncture; the outcome was excellent. However, there are reports that only $25 \%$ of dogs recover from lameness after the conservative treatment $[1,2,18]$. Thus, if there is no improvement of clinical signs after four weeks, surgery should be performed [13].

The femoral head and neck ostectomy was the surgical procedure performed in 42 hind limbs in the present study. The procedure is used to provide pain relief and to reduce the signs of lameness $[2,8,9,12,14]$. Although good results are expected from this procedure, some dogs may experience mild intermittent lameness [13]. As for the present study, $42.85 \%$ of the dogs had total functional recovery of the hind limb, but $26.19 \%$ required physiotherapy and rehabilitation. In two dogs another procedure was necessary to remove the remaining portion of the femoral neck. These cases reflect the importance of the surgical technique, since bone-on-bone contact can induce postoperative pain [13]. The micro total hip replacement was another treatment option successfully used in seven dogs with avascular necrosis of the femoral head [5].

\section{CONCLUSIONS}

The population assessed in the present study was composed of small size dogs, mean body mass 4.33 $\mathrm{kg}$, no sex predilection, mostly presenting unilateral lesions and higher Grade 2 and Grade 5 radiographic lesion frequency.

Acknowledgements. We are grateful to CNPq (National Council for Scientific and Technological Development).

Ethical approval. FMVZ/Botucatu-SP - CEUA n ${ }^{\circ}$ 55/2013.

Declaration of interest. The authors report no conflicts of interest. The authors alone are responsible for the content and writing of the paper. 


\section{REFERENCES}

1 Demko J. \& McLaughlin R. 2005. Developmental orthopedic disease. Veterinary Clinics of North America: Small Animal Practice. 35(5): 1111-1135.

2 Gambardella P.C. 1993. Legg-Calvé-Perthes disease in dogs. In: Bojrab M.J., Smeak D.D. \& Bloomberg M.S. (Eds). Disease mechanisms in small animal surgery. Philadelphia: Lea \& Febiger, pp.804-807.

3 Gibson K.L., Lewis D.D \& Pechman R.D. 1990. Use of external coaptation for the treatment of avascular necrosis of the femoral head in a dog. Journal of American Veterinary Medical Association. 197(7): 868-870.

4 Isola M., Zotti A., Carnier P., Baroni E. \& Busetto R. 2005. Dual-energy x-ray absorptiometry in canine Legg-CalvéPerthes disease. Journal of Veterinary Medicine Series A. 52(8): 407-410.

5 Jankovits D.A., Liska W.D. \& Kalis R.H. 2012. Treatment of avascular necrosis of the femoral head in small dogs with micro total hip replacement. Veterinary Surgery 41(1): 143-147.

6 Jones D.G.C. 1985. Conditions of the canine hip joint. The British Veterinary Journal. 141(6): 554-563.

7 Kemp H.B.S. 1986. Perthes' disease in rabbits and puppies. Clinical Orthopaedics and Related Research. 209(1): 139-159.

8 Lee R. \& Fry P.D. 1969. Some observations on the occurrence of Legg-Calvé-Perthes disease (coxaplana) in the dog, and evaluation of excision arthroplasty as a method of treatment. Journal of Small Animal Practice. 10(5): 309-317.

9 Lika E., Gjino P., Belegu M., Duro S., Dimco E., Sherko E. \& Turmalaj L. 2012. Retrospective study of the treatment of aseptic necrosis of the femoral head in dogs. Journal of Animal and Veterinary Advanves. 11(16): 2930-2933.

10 Mickelson M.R., Mccurnin D.M., Awbrey B.J., Maynard J.A. \& Martin R.K. 1981. Legg-Calvé-Perthes disease in dogs: A comparison to human Legg-Calvé-Perthes disease. Clinical Orthopaedics and Related Research. 157(1): 287-300.

11 Nadel S.N., Debatin J.F., Richardson W.J., Hedlund L.W., Senft C., Rizk W.S., Malizos K.N., Stahl D.L. \& Martinez S. 1992. Detection of acute avascular necrosis of the femoral head in dogs: dynamic contrast-enhanced MR imaging vs Spin-echo and STIR sequences. American Journal of Roentgenology. 159(6): 1255-1261.

12 Nebzydoski J.A. 1982. Ischemic necrosis of the femoral head in dogs: a review. Veterinary Medicine \& Small Animal Clinician. 77(4): 631-636.

13 Piek C.J., Hazewinkel H.A.W., Wolvekamp W.T.C., Nap R.C. \& Mey B.P. 1996. Long term follow-up of avascular necrosis of the femoral head in the dog. Journal o Small Animal Practice. 37(1): 12-18.

14 DeCamp C.E., Johnston S.A., Déjardin L.M. \& Schaefer S.L. 2016. The hip joint. In: Piermattei D.L. (Ed). Brinker, Piermattei, and Flo's handbook of small animal orthopedics and fracture repair. 5th edn. St. Louis: Elsevier, pp.468517.

15 Robinson R. 1992. Legg-Calvé-Perthes disease in dogs: genetic aetiology. Journal of Small Animal Practice. 33(6): 275-276.

16 Singleton W.B. \& Jones E.L. 1979. The experimental induction of subclinical Perthes' disease in the puppy following arthrotomy and intracapsular tamponade. Journal of Comparative Pathology. 89(1): 57-71.

17 Stockman M. 1982. Inheritable defects in dogs: 1. In Practice. 4(6): 170-175.

18 Towle H.A. \& Breuer G.J. 2012. Miscellaneous orthopedic conditions. In: Tobias K.M. \& Johnston S.A. (Eds). Veterinary surgery small animal. St. Louis: Elsevier Saunders, pp.1112-1126.

19 Vasseur P.B., Foley P., Stevenson S. \& Heitter D. 1989. Mode of inheritance of Perthes's disease in Manchester terriers. Clinical Orthopaedics and Related Research. 244(1): 281-92. 